\title{
ANDRÉ MALRAUX: ROMAN, HISTOIRE ET QUÊTE IDENTITAIRE
}

\author{
JOSEPH ABAH ATANGANA
}

Université de Yaoundé I, Ecole Normale Supérieure

\begin{abstract}
Résumé
Les rapports entre l'histoire et la création littéraire ont souvent été difficiles à préciser car les deux ont toujours cohabité. Compte tenu de cette cohabitation, il importe de poser la question de savoir si le romancier historien est un historien. Cet essai n'a pas l'intention de faire une étude systématiquement comparative entre L'Espoir d'André Malraux et les faits historiques, mais de mettre un accent particulier sur la pression de l'histoire sur le roman. Le romancier accapare l'histoire au moyen de différentes techniques pour en proposer une lecture personnelle. L'histoire se trouve pour ainsi dire phagocytée non pas au sens biologique strict du terme qui stipulerait sa capture, sa destruction et sa digestion d'où sa désagrégation totale, mais au sens symbolique et littéraire, qui infère son exploitation par la fiction, c'est-à-dire son intégration et son utilisation à des fins esthétiques.
\end{abstract}

Mots clés: histoire, fiction, réalité, création littéraire, identitaire.

\begin{abstract}
Relationships between history and literary creation have often been difficult to determine since both has always been interconnected. Having regard to this coexistence, it is important to ask whether the novelist-historian is a historian. This essay does not intend to make a systematic comparative study between L'Espoir written by André Malraux and the historical facts, but to focus on the influence of history on the novel. The novelist seizes history through various techniques to offer a personal interpretation. History is therefore absorbed not in the biological sense of the word which may mean its capture, destruction and digestion, that is its complete dissolution, but in the symbolic and literary sense which entails its exploitation by fiction that is its integration and use for aesthetical.
\end{abstract}

Keywords: history, fiction, reality, literary creation, identity.

\section{INTRODUCTION}

En Europe et précisément en Allemagne, en Italie et en Espagne, les années trente sont marquées par l'avènement et la menace du fascisme. L'histoire des relations internationales européennes est dominée de 1933 à 1939 par les initiatives d'Adolf Hitler. Celui-ci ne dissimule pas sa volonté d'expansion. Ses ambitions démesurées basculent inéluctablement l'Europe vers la guerre. «Depuis qu'Hitler est au pouvoir,

1 Université de Yaoundé I, Ecole Normale Supérieure. Correo: abarise2002@yahoo.fr. Recibido: 12-122011. Aceptado: 29-02-2012. 
la tragédie a gagné l'Europe.» (Raimond, 1976: 218). À partir de 1933, les espoirs d'un règlement pacifique des différends internationaux s'estompent et disparaissent. Le monde change instantanément en une véritable jungle où la raison du plus fort devient incontestablement la meilleure. On ne parle plus qu'en termes d'attentat, d'attaque, de frappe, d'invasion, d'annexion; en guise de réplique, les uns et les autres agissent en coalition et par conséquent il ne s'agira que de triomphe ou de reddition. L'affrontement armé devient inévitable. «L'armée espagnole, affirme Manuel, on n'a jamais entendu parler d'elle qu'en cas de défaites. (Malraux, 1937: 106). ${ }^{2}$

Précisons d'entrée de jeu qu'en février 1936, le Front populaire vient de remporter les élections en Espagne. La jeune République se débat quelque temps entre les attentats phalangistes et les grèves antifascistes. Le 17 juillet de la même année, les troupes du Maroc espagnol se soulèvent d'un seul coup sous le commandement du Général Franco. L'insurrection touche bientôt l'Espagne entière. Le gouvernement décide rapidement d'armer le peuple contre l'armée:

À une heure du matin, déclare le narrateur, le gouvernement avait enfin décidé de distribuer les armes au peuple; à trois heures, la carte syndicale donnait droit aux armes. (Malraux, 1937: 11.)

Chose promise, chose due: «La distribution des armes prise commençait. " (Malraux, 1937: 52.) C'est le début d'une guerre intestine qui va durer près de trois ans. La Guerre civile espagnole est donc l'un des problèmes internationaux de 1933 à 1947.

La guerre qui déchire l'Espagne de juillet 1936 à mars 1939 est l'un des événements majeurs d'avant la Seconde Guerre mondiale, étant entendu que les Fascistes y ont testé la puissance de frappe de leur armement. Il y a soixante-seize ans (1936-2012) lorsque cette guerre éclate qui engage écrivains et journalistes entre autres. André Malraux (1901-1976) ${ }^{3}$ va y prendre part aux côtés des Républicains et cette douloureuse expérience va produire aussitôt $L$ 'Espoir. Le Français se jette à corps perdu dans le combat de la République espagnole traquée par le fascisme. Il partage le point de vue de Boisdeffre:

Que chaque homme est lié au monde, que chaque parole a des retentissements, que chaque silence peut être complice. (Boisdeffre, 1963: 3).

Selon Garcia, «l'action ne se pense qu'en termes d'action.» (Malraux, 1937: 250.) Pour justifier son engagement, Malraux, exaspéré, affirme:

Depuis dix ans, le fascisme allemand étend sur l'Europe ses grandes ailes noires. Le fascisme allemand nous montre que nous sommes face à la guerre. Nous devons faire notre possible pour qu' elle n'ait pas lieu: mais nous avons affaire à des sourds, nous savons qu'ils n'entendent pas! À la menace, répondons par la menace, et sachons nous tourner vers Moscou, vers l'Armée rouge! (Biet et al., 1987:62).

2 André Malraux, L'Espoir, Paris, Gallimard, coll. «Folio», 1937. Sauf indication contraire, c'est à cette édition que se réfèrent toutes nos citations.

3 Cherche dans l'engagement politique et dans l'art les moyens de lutter contre la corruption du temps et l'instinct de la mort. 
Malraux prône sans ambages une action sang contre sang et contre la terreur hitlérienne. Il réussira à convaincre une bonne partie du monde de venir à la rescousse de l'Espagne dans le but de sauver la démocratie foulée aux pieds par ce qui peut s'appeler aujourd'hui «l'axe du mal» et ce qu'André Brincourt appelle «les puissances du mal qui menacent l'homme de notre temps». (Brincourt, 1986: 56.) Aux côtés des Républicains, il allait donc vivre de près la tragédie espagnole. Ainsi, Malraux était bien parti pour être l'historien de la guerre civile espagnole et son roman se prêtait beaucoup plus à une œuvre d'historien qu'à celle d'un romancier.

Dans $L^{\prime}$ Espoir, la guerre inspire Malraux. La première partie du roman se déroule sur fond d'une immense exaltation. Le gouvernement républicain a armé les milices populaires pour combattre le coup d'État des généraux fascistes qui s'appuient sur l'armée maure. Communistes, anarchistes, socialistes, une partie de l'armée et de la garde civile espagnoles s'allient pour la cause commune. Tandis que les Républicains sont victorieux à Madrid, à Barcelone et dans la Sierra, où les opérations sont dirigées par le communiste Manuel, l'escadrille commandée par Magnin résiste aux avions ennemis à Medellin. Face à l'armée du Général Franco et à l'arsenal de tanks et des avions que lui envoient ses alliés italiens et allemands, l'enthousiasme et le courage ne suffisent plus, comme le démontre l'échec du siège et de l'attaque de l'Alcazar de Tolède.

S'agissant de la deuxième partie du roman, l'organisation du combat, reprise en main par les communistes, permet de maîtriser la déroute de Tolède, de mettre en fuite les Maures au-delà du Manzanares, et assure la victoire du parc de l'Ouest à Madrid et de la route de Corogne.

Quant à la troisième et dernière partie, l'aviation combat sur le front du Levant. Le 15 février, l'escadrille de Magnin est bombardée par les avions ennemis dans la Sierra de Teruel. Les aviateurs blessés sont transportés sur des civières par les paysans de Linares. Le roman s'achève sur l'avancée des bataillons de Manuel au cours de la bataille de Guadalajara, soutenus par l'aviation républicaine, qui dispose maintenant d'avions soviétiques.

En réalité, l'Histoire et la fiction sont les points de suture de l'écriture de Malraux, mais ils sont en même temps des voies pour pénétrer le texte et le questionner.

Comment et pourquoi Malraux opère-t-il une transformation fictionnelle sur l'Histoire pour créer et animer sa société fictive dans son roman?

L'écriture particulière et particularisante dudit romancier est-elle spécifiquement une affirmation de l'identité de l'auteur?

Dans le cadre des rapports interdisciplinaires entre l'Histoire et la fiction, l'hypertextualité et la veine thématique permettront de montrer que le support littéraire est fortement nourri aux sources de l'Histoire et qu'il affiche l'interaction agissante entre l'Histoire et la fiction.

Pour ne pas avoir une vue étriquée de la question, des rapprochements avec Pour qui sonne le glas seront indispensables, les noms de Ernest Hemingway et de Malraux 
étant indissociables en parlant de la guerre civile espagnole. Comme L'Espoir, Pour qui sonne le glas reflète aussi l'évolution des premiers mois de la guerre civile entre Franco et les Républicains espagnols, auprès desquels les deux romanciers combattent.

Le présent essai sera stratifié en trois parties. La première s'articulera autour de la pression de l'Histoire sur le roman. La deuxième s'intéressera à l'esthétisation de l'Histoire. Elle montrera comment l'Histoire se lit dans le prisme artistique et esthétique du roman. En effet, ici l'exploitation fictionnelle de l'histoire se fait dans le but de l'expression identitaire, contenu de la troisième partie. Etant entendu que:

Avec les deux «aventures indochinoises», vient le temps des combats sérieux, celui des romans épiques, lyriques, en un mot: révolutionnaires. Ils sont écrits par celui que Drieu la Rochelle appelle «l'homme nouveau». Ils plongent leur lecteur au cœur des tourmentes politiques et sociales, mais posent aussi, dans toute sa modernité, la question de l'identité personnelle. (Larrat, 2001: 5.)

\section{LE POIDS ET LA PRESSION DE L'HISTOIRE}

Pourquoi lit-on L'Espoir ? Certainement à cause de l'intérêt historique et politique de l'événement. La guerre civile espagnole, par ses causes, ses conséquences, sa date, les passions qu'elle a soulevées, a profondément marqué la conscience des contemporains.

Le roman d'André Malraux a pour cadre la Guerre civile espagnole. Il s'ouvre sur une précision du lieu, du moment et de la saison. Le premier chapitre situe le lecteur au cœur de Madrid en ces termes:

Un chahut de camions chargés de fusils couvrait Madrid tendue dans la nuit d'été. (Malraux, 1937: 11.)

Sur le plan géographique, les noms des localités du roman se trouvent effectivement en Espagne. Parlant du cadre spatial, il est évident que Malraux ne l'a pas improvisé. L'exactitude en est rigoureuse. L'évocation des villes telles que: Tolède, Saragosse, Valladolid, Séville, Ségovie, Madrid, Barcelone, etc., plonge le lecteur dans la carte de l'Espagne actuelle. À la réalité des villes correspond aussi la réalité des noms de personnes liés à l'histoire de la Guerre civile espagnole : Franco, Queipo de Llano $^{4}$.

L'Espagne est le théâtre des hostilités même si Malraux y mêle avec les convenances de sa liberté d'écrivain des éléments imaginaires. Ainsi, avec Stendhal, L'Espoir semble accomplir la fameuse définition citée dans Le Rouge et le Noir «le roman est un miroir qui se promène sur une grande route.» (Stendhal, 1988: 208). Autrement dit, Malraux brosse une fresque historique et sociale de l'Espagne. Une manière de dire que:

On ne peut guère concevoir de «roman pur» où tout serait totalement fabriqué, détaché de la réalité. (Bourneuf et Ouellet, 1972: 25).

4 Général espagnol qui fut l'un des principaux lieutenants de Franco pendant la guerre civile. 
Dans L'Espoir, il est question des problèmes que pose aux Républicains la conduite de la guerre. Toutefois, on peut constater l'omission relative à la représentation du vécu du quotidien du peuple espagnol.

Malraux connaît l'espagnol et il le parle. Dans son roman, les mots espagnols insérés dans le texte français sont bien autre chose qu'un artifice littéraire. Il cite un sonnet de l'écrivain espagnol Quevedo: «¿Qué pretende el temor desacordado de la que a rescatar piadosa viene espíritu en meserias añudado?» (Malraux, 1937: 375.) Ils ont la force de la vie et de la vérité. Ils expriment aussi une violence que sans doute le romancier désespérait de traduire.

À la lecture de L'Espoir, la toute première des remarques qui s'impose est que Malraux, loin de vouloir faire œuvre d'historien, s'intéresse à la guerre d'Espagne pour des raisons personnelles.

Deux fois, j'ai vu le peuple d'Espagne, déclare le narrateur, cette guerre est sa guerre, quoi qu'il arrive ; et je resterai avec lui là où il est. (Malraux, 1937: 362.)

La guerre d'Espagne était pour Malraux la guerre et l'Espagne. C'est dire qu'elle représentait un double intérêt pour le romancier : l'amour qu'il vouait à ce pays et la guerre qui le rongeait et le ravageait.

Un homme ne peut tolérer l'indignité de ce qui se passe ici, confie le capitaine Mercery. Je suis au service de l'Espagne! Dans n'importe quelle fonction: mais au service de l'Espagne. Il faut en finir avec le fascisme. (Malraux, 1937: 94.)

Une attitude que partage entièrement l'équipage du Marat:

Nous ne sommes venus ici pour aucune aventure. Révolutionnaires sans parti, socialistes ou communistes résolus à défendre l'Espagne, nous combattrons dans les conditions les plus efficaces, quelles qu'elles soient. Vive la liberté du peuple espagnol. (Malraux, 1937: 235-236).

La mission de l'homme étant, selon les mots de Garcia, «de transformer en conscience une expérience aussi large que possible.» (Malraux, 1937:321.) Janet Flanner remarque que:

Le 18 juillet, la guerre civile espagnole éclata. Deux jours plus tard, Malraux était en Espagne. (Langlois, 1973: 93.)

Malraux rencontra beaucoup dejournalistes et d'écrivains qui convergeaient vers le théâtre des opérations. Certains étaient là pour des raisons purement personnelles, ou du fait de leurs convictions, ou encore pour enquêter, comme Antoine de SaintExupéry pour L'Intransigeant. D'autres écrivains défendaient la causa à titre individuel ou professaient d'autres opinions. André Malraux avait joué un rôle décisif et franc en fournissant des avions français à la causa ou en organisant l'escadrille aérienne de la Brigade Internationale à Alcantarilla.

La guerre d'Espagne éveilla les pulsions humanitaires de Malraux, mais elle aiguillonna aussi son sens de la compétition littéraire avec la publication en 1937 de L'Espoir, bien avant Pour qui sonne le glas d'Ernest Hemingway qui date de 1940.

5 La cause commune. 
Pour Guernico, «nous, écrivains chrétiens, nous avons peut-être plus de devoirs que d'autres». (Malraux, 1937: 362.) Selon Malcom Cowley:

Hemingway projetait d'écrire un roman sur la guerre civile espagnole depuis sa première visite à Madrid après la déclaration des hostilités. Il avait rencontré André Malraux, qui à ce moment faisait partie de l'aviation loyaliste et ils s'étaient entendus mystérieusement pour se partager la guerre ; tout ce qui s'était passé jusqu'à la défaite italienne de Brihuega en avril 1937 appartiendrait à Malraux. Mais celui-ci travailla rapidement - trop rapidement au dire d'Hemingway - et L'Espoir parut en décembre de la même année. (Lacasse, 1977: 63.)

En fait, Malraux passe d'une guerre à une autre. Après l'épisode de la Chine, il se rend tout de suite en Espagne. Jean-Claude Larrat précise que:

Le mythe du combattant révolutionnaire, qu'il avait laissé se développer à son retour d'Indochine, devenait, en Espagne, une réalité. ( Larrat, 2001: 222).

Denis Marion fait remarquer que «L'Espoir est un récit chronologique qui commence le 18 août 1936 et qui s'achève en juillet 1937.»(Marion, 1996: 43). Dès le 18 juillet 1936, trois jours seulement après le début du putsch des généraux espagnols, Malraux part pour l'Espagne, où il crée et dirige l'escadrille España pour soutenir la cause républicaine. Le combattant cède alors la place à l'écrivain qui se met sur-lechamp à la rédaction de L'Espoir, nourri de l'expérience exceptionnelle qu'il vient de vivre.

À l'espace confiné de La Condition humaine - qui se réduit, pour la quasitotalité du roman, à la ville de Shanghaï - succède ici l'éclatement de la topographie romanesque. De Barcelone à Tolède (première partie) ou de Málaga à Guadalajara (troisième partie), les actions s'éparpillent dans l'ensemble du pays ibérique. Ce roman emprunte à la fois au récit historique et journalistique mais l'importance est accordée à l'événement (combat) qui est ici situé et daté.

On comprend facilement que l'Espagne est l'un des plus grands chapitres de la vie de Malraux. Évocation de son expérience espagnole, L'Espoir est aussi l'épopée de la guerre civile et une œuvre fraternelle où passe un souffle tolstoïen:

$\mathrm{Au}$-dessus d'eux, en arrière, la lune, qu'ils ne voyaient pas, éclairait l'aluminium des ailes. Leclerc reposa sa thermos : aucun geste humain n'était plus à la mesure des choses ; bien loin de ce cadran de guerre seul éclairé jusqu'à des lieues, l'euphorie qui suit tout combat se perdait dans une sérénité géologique, dans l'accord de la lune et de ce métal pâle qui luisait comme les pierres brillent pour des millénaires sur les astres morts. (Malraux, 1937: 256-257).

Son livre paru, Malraux ne tourne pas la page de l'Espagne et veut maintenant réaliser un film. Le tournage de Sierra de Teruel - qui n'est pas une adaptation du roman, mais seulement de quelques-uns de ses chapitres - commença en juillet 1938 à Barcelone. Il devait se poursuivre en d'autres lieux de Catalogne, enfin à Villefranchede-Rouergue où, quelques années plus tard, Malraux, prisonnier de la Wehrmacht, sera logé pour une nuit mémorable.

En associant les localités et les noms d'origine espagnole, Malraux veut démontrer la véracité de son récit en le situant dans un contexte et dans un lieu précis: la Guerre civile espagnole. Preuve: 
(...) qu'une scène dans un roman est soumise à des principes d'unité - lieu, temps, action - et nous le verrons, dans une certaine mesure, de point de vue. L'auteur indique avec plus ou moins de précision le cadre physique. (Bourneuf et Ouellet, $1972: 61$.)

L'Espoir est donc sans doute le récit romancé de la participation rageuse de Malraux à la guerre civile espagnole. En effet, dès le 21 juillet, il sillonne Madrid, Barcelone et met sur pied l'escadrille España, car il a très vite compris qu'une aviation, même embryonnaire, est indispensable à la République dans les premières semaines de la guerre. À la tête de cette escadrille, il combat effectivement d'août 1936 à février 1937. À bien des égards, son roman est un reportage, même s'il transpose la réalité des événements qu'il a vécus et confère un souffle épique à ces combats:

L'enquête, dit Jean Lacouture, auprès des témoins les moins récusables, et la lecture des trois ou quatre meilleures histoires de la guerre d'Espagne, recoupent les principales indications du roman en ce qui touche à la vie et aux activités de l'espadrille. (Stéphane, 1996: 74.)

Malraux était soucieux de témoigner en faveur de ses camarades de combat, d'évoquer l'élan fraternel de tout un peuple. Magnin, le patron de l'escadrille dans la fiction n'est pas tout à fait Malraux ; en revanche, on trouverait des aspects de Malraux dans les autres personnages du livre : Garcia, Scali, Manuel, Hernández. En dehors de l'activité de son escadrille, Malraux relate les combats qui ont commencé en juillet 1936 jusqu'à la victoire républicaine de Guadalajara, en mars 1937. Il décrit la résistance populaire de Madrid, de Barcelone, où les travailleurs sont armés par les syndicats; il raconte le siège de l'Alcazar à Tolède et la débandade des troupes républicaines non organisées; puis le bombardement de Madrid, à la fin d'octobre, effectué pour démoraliser la population; et les combats aériens pour la défense de Madrid.

La guerre civile espagnole n'est donc qu'un repère historique pour le roman. Une déclaration de Malraux, datée de 1935, pourrait servir d'exergue, en épigraphe à L'Espoir: "une œuvre d'art est un objet, mais aussi une rencontre avec l'histoire.» (Fitch, 1964: 74.) Il apparaît ici que Malraux subit le poids et la pression de l'Histoire pour rédiger L'Espoir. Il produit logiquement un roman assiégé par l'Histoire.

\section{L'HISTORICISATION DE LA FICTION}

La réécriture de l'Histoire ou l'historisation de la fiction vise à montrer que l'Histoire dont il est question ici n'est pas une simple reproduction de la réalité mais qu'elle est enrichie de la fantasmation de l'auteur de La Voie royale. Autrement dit, L'Espoir repose d'abord sur la puissance du verbe, l'élégance de la langue et surtout du parti pris de Malraux.

Gustave Flaubert fait remarquer que «le style est à lui tout seul une manière de voir les choses.» (Labesse, 1995: 110.) Il apparaît assez nettement que Malraux présente des événements qui se sont réellement déroulés en Espagne, mais comme artiste, il fait preuve d'une très grande capacité de réécrire ces récits selon son tempérament ou sa vision du monde. C'est en quelque sorte l'exaltation du lyrisme. À suivre de près ces récits et lorsqu'on les compare à ce qui s'est passé dans la réalité, il se dégage 
l'impression qu'ils sont bien différents et pourtant ils ont la même origine: la guerre civile espagnole.

En réalité, nous pouvons dire que nous sommes en face des mêmes événements mais avec un regard différent ou particulier. Celui d'un romancier qui réécrit la guerre ou réécrit purement le passé ou la Grande Histoire et instruit non seulement sur le réel mais aussi et surtout sur la fiction ou même la fantasmation du romancier.

Le constat qui se dégage est que la fiction ne peut rivaliser avec la réalité. La fiction est, comme on le sait, imaginaire ou irréelle tandis que la réalité est ce qui existe, ce qui est authentique. La fiction et la réalité peuvent cependant se côtoyer allègrement dans le but de donner au récit les allures de vraisemblance ou d'authenticité. Ainsi le roman devient-il un «mentir vrai» selon l'expression de Louis Aragon. Ceci n'est pas un roman historique, affirme-t-il en substance, mais une impudente exploitation de l'histoire dont j'ai fait un marchepied à la fiction, un passeport pour le roman. Pour Aragon:

Ce qui est menti dans le roman libère l'écrivain, lui permet de montrer le réel dans sa nudité. Ce qui est menti dans le roman est l'ombre sans quoi vous ne verriez pas la lumière. Il s'agirait pour nos écrivains de se résigner à ne plus faire concurrence à la vie. Étant entendu que l'essentiel, dans la vie, n'est jamais exprimé. Car il faut reconnaître avant tout que l'art du roman est une transposition du réel et non une reproduction servile du réel. (Beaumarchais et al, 2001: 41.)

Dans ce sens, François Mauriac amène à accepter humblement que «les personnages romanesques sont une image transposée et stylisée.» (Mauriac, 1972 : 278.) Pour lui:

(...) acceptons d'atteindre le vrai que par réfraction ou par écho. Il faut se résigner en réalité aux conventions et aux mensonges de l'art romanesque. (Mauriac, 1972 : 278.)

En clair, Malraux crée un monde à lui. Le monde du XXème siècle qu'il peint, mais vu par lui, agrandi, exagéré, dramatisé. Il expose sa vision du monde à travers l'observation du réel. Pour André Rousseaux, le talent de Malraux:

(...) est l'aptitude à fournir des truquages littéraires des aventures qu'il a voulu vivre. Le démon de Malraux aime à lui faire croire à des choses qu'il aurait voulu faire. (Gaillard, 1970: 74.)

La réalité prend des proportions gigantesques. La vue se transforme en seconde vue. Ce qui, dans l'univers réel, était contingence, devient signe et symbole. Tout devient signifiant dans l'univers de Malraux.

Le choix d'un événement historique comme matière romanesque est déjà très significatif en lui-même. C'est que le romancier accorde une très grande importance à l'Histoire. Il nous livre sa propre conception de l'Histoire. Pour Malraux, c'est une histoire négative, accablante et écrasante représentée par les atrocités et les horreurs de la guerre qui révèlent à l'homme sa condition. Aussi refuse-t-il de la sacraliser et légitimer sa toute puissance. Il procède plutôt à une mise en accusation de la guerre.

Bien plus, pour le romancier français, c'est une histoire collective et commune. «On est tous dans le même bain» (Hemingway, 1944: 324.) avance Robert Jordan. Cette phrase très représentative des années 30 à 45, se trouve aussi dans La Peste où Albert 
Camus, en temps d'épidémie, note «il n'y avait plus de destin individuel.» (Camus, 1947: 98). Et c'est à juste titre que Pol Gaillard affirme:

Par lui, «tous les hommes de bonne volonté» en Europe se trouvèrent intimement liés à la lutte que le peuple d'Espagne était en train d'entreprendre contre le fascisme. Malraux vint non pas en ambassadeur de paix, mais en guerrier, pour prendre sa place (comme dit un de ses amis espagnols) «dans la ligne de combat... armé des armes indomptables» de son intelligence, de son énergie et de sa noblesse de sentiments. Pendant presque trois ans il mit tous ces talents au service de la République. (Gaillard, 1970: 74.)

En son temps, l'écrivain irlandais Oscar Wilde, adepte de l'esthétisme, soutenait que le seul et unique devoir que nous avons avec l'histoire, c'est de la réécrire ou de la reconstituer. Refaire ou reconstruire l'histoire semble donc être la raison d'être de L'Espoir étant donné que le romancier se sert de celle-ci comme prêt-à-porter dans l'élaboration de son roman.

Tomás Eloy Martínez ajoute que restituer ou recomposer fidèlement l'histoire est presque chose impossible; c'est pour cela qu'il déclare de façon pessimiste que :

Tout récit est, par définition infidèle. La réalité [...] ne peut se raconter ni se répéter. La seule chose qu'on puisse faire avec la réalité, c'est la réinventer.» Si l'histoire relève des genres littéraires, «pourquoi la priver de l'imagination, de l'extravagance, de l'exagération, de l'échec, qui sont la matière première de la littérature? (Fuentes, 1997: 183.)

Pour Eloy Martínez, l'écrivain ou le romancier a pour objectif de repenser l'histoire, étant entendu qu'il ne peut pas reproduire ou reconstituer exactement ce qui s'est réellement et exactement passé. Il ne peut que reconstruire la réalité à sa manière, à sa façon ou à sa guise, selon son imagination ou sa fantasmation: c'est le parti pris de l'écrivain qui regarde le monde à travers son tempérament. Un monde qui devient, du fait de la présence de l'écrivain, subjectif, parcellaire, fragmentaire et partial. Manifestement, il y a difficulté à l'impartialité. L'art, et particulièrement la littérature, sont choses humaines et il semble difficile que l'œuvre littéraire exclue toute partialité, c'est-à-dire en somme tout parti pris, toute marque de l'homme sur le réel.

Il s'agirait de reconnaître que l'art est, par définition, arbitraire et que même en n'atteignant pas le réel dans toute sa complexité, il est tout de même possible d'atteindre des aspects de la vérité humaine. Il faudrait donc reconnaître avec Mauriac que «l'art du roman est, avant tout, une transposition du réel et non une reproduction du réel.» (Mauriac, 1972 : 278.) il va de soi que pour de Zola, «une œuvre d'art est un coin de la création vu à travers un tempérament.» (Beaumarchais et al., 2001: 2065.) L'artiste, affirme le même Zola, est celui qui veut «vivre tout haut.» (Beaumarchais et al., 2001: 2065.) Au bout du compte, Malraux essaie de représenter l'ensemble de la société de son temps, mais il ne peut s'empêcher de lui imprimer ses marques. On note, par exemple, que les héros de Malraux sont doués, comme leur créateur, d'énergie farouche, de volonté de puissance. Bref, ils portent tous la marque du tempérament de leur créateur.

Malraux aide à saisir la vie, l'âme, la physionomie des choses et des êtres dans une écriture où se mêlent inextricablement le réel et l'imaginaire. On ne pense pas 
assez que le roman qui serre la réalité du plus près possible est déjà tout de même menteur par cela seulement que les héros s'expliquent et se racontent.

Et cependant, grâce à cette falsification, de grandes vérités partielles ont été atteintes. Pour Mauriac, ces:

(...) personnages fictifs et irréels aident à mieux nous connaître et à prendre conscience de nousmêmes. Ce ne sont pas les héros de roman qui doivent servilement être comme dans la vie, ce sont au contraire, les êtres vivants qui doivent peu à peu se conformer aux leçons que dégagent les analyses des grands romanciers. (Mauriac, 1972 : 277.)

Aussi vivante qu'apparaisse une créature romanesque, il y a toujours en elle un sentiment, une passion que l'art du romancier hypertrophie pour que nous soyons mieux à même de l'étudier, aussi vivants que ces héros apparaissent:

(...) ils ont toujours une signification, leur destinée comporte une leçon, une morale s'en dégage qui ne se trouve jamais dans une destinée réelle toujours contradictoire et confuse. (Mauriac, 1972 : 204.)

Malraux tout comme l'historien, s'approprie la mémoire de l'Histoire. Il y a donc une sorte de décalage entre ce qui a été vécu et ce qui est rapporté ; lisible dans la partie de création du romancier.

S'il faut faire coexister l'Histoire et la fiction, il apparaît par conséquent que cette dernière déborde l'Histoire, la contient, la particularise en même temps qu'elle la généralise. Malraux re-crée l'Histoire en créant son histoire, sa diégèse. Il établit par ce fait un rapport mimétique entre L'Espoir et l'Histoire. Ces aspects de l'analyse sont évoqués par Yves Le Pellec et Marcienne Rocard dans Caliban consacré au roman historique. À propos des spécialistes du roman historique, ils notent que :

[...] Ils privilégient les interstices de l'histoire officielle, les passages secrets et les souterrains, tous les lieux que la doxa historique n'a guère explorés et où, à l'écart des têtes couronnées et en marge des grands événements, pourront se déployer les destins personnels des héros imaginaires. Car le roman particularise l'histoire, en même temps qu' il l'universalise. D'une part il la détourne de sa globalité pour montrer son incidence sur des existences individuelles, d'autre part il la dégage de sa temporalité pour la situer dans une réflexion sur la condition humaine éternelle. D'être intégrée dans le roman, l'histoire se voit en quelque sorte « déshistoricisée » et resémantisée, car elle devient composante esthétique d'une structuration formelle, et unité de sens en relation avec tous les autres éléments de l'isotopie générale délimitée par la clôture du texte. (Le Pellec et Rocard, 1991: 6.)

Il en ressort que la réécriture de l'Histoire et son esthétisation lui ôtent sa substance scientifique pour la poser comme un possible de l'imagination. Il est donc clair que la frontière entre l'Histoire et la fiction est une «frontière mouvante » (Le Pellec et Rocard, 1991: 8.), jamais délimitée et difficile à indiquer. La récupération thématique que fait Malraux amène le lecteur dans les abysses de l'Histoire et établit un pont entre son texte et le hors-texte.

De tout ce qui précède, il apparaît que des personnages historiques, mythologiques, allégoriques ou sociaux, dans le sens de Philippe Hamon : 
(...) tous renvoient à un sens plein et fixe, immobilisé par une culture, à des rôles, des programmes, et des emplois stéréotypés. (Barthes et al, 1977:122).

Intégrés à un énoncé:

(...) ils servent essentiellement $\mathrm{d}^{\prime}$ ancrage référentiel et participent à la désignation automatique du héros et sont les marques de la présence en texte de l'auteur. (Barthes et al, 1977: 122).

En outre, il ne s'agit pas pour Malraux de reconstitution de l'Histoire mais plutôt de reportage faute de recul par rapport à l'événement. Même s'il n'y a pas reconstitution de l'Histoire, il y a par contre construction des personnages aux destins divers contrastant avec la vie des personnages historiques auxquels ils renvoient. Ici, nous sommes très loin du travail d'un historien. Le romancier entraîne le lecteur dans son monde à lui. Il laisse exploser ses talents d'artiste, ses dons de créateur. Dans ce qui est supposé être l'histoire de la guerre civile d'Espagne, il réussit à insérer l'histoire de sa vie, de sa philosophie et de sa vision du monde. Son héros, comme dans la littérature romantique, n'est que la projection sur scène de l'auteur resté dans la coulisse. À travers son roman, Malraux réussit à rendre compte des inquiétudes, de l'angoisse et des réalités de la guerre d'Espagne.

Tout comme la reconstitution, l'histoire reste fictive: comment comprendre, par exemple, que Malraux réduise toute la guerre civile espagnole à Tolède? Tandis que le reporter de guerre couvrait tout le pays en faisant des reportages objectifs, l'auteur Des Conquérants ne s'attachait qu'à une portion du pays, ne découvrant que lui-même et ne traitant que des problèmes inhérents à la condition humaine: l'absurde, la mort, l'habitude, le destin, la violence, etc. À l'exemple de Malraux, Hemingway situe le point culminant de son roman autour du pont dont Robert Jordan a pour mission de faire sauter. Le succès du héros dépend entièrement de l'issue de la bataille qui s'y prépare. Le sort de la guerre entre Républicains et Fascistes semble étroitement lié à ce même pont.

En analysant L'Espoir, le titre ne se réfère pas directement à la guerre d'Espagne. Pourquoi Malraux a-t-il intitulé son roman L'Espoir alors qu'au moment de sa publication, la défaite du camp républicain qu'il défendait était certaine? Pour le narrateur:

Le monde entier, à cette minute coulait dans un seul sens...ces gens accroupis ou couchés suaient l'angoisse, dans l'immobilité comme dans la fuite. Entre eux, la masse passive des sanslogis continuait vers Almeria sa migration désespérée. (Malraux, 1937: 508.)

Il est vrai que son roman se termine sur la victoire républicaine de Guadalajara, mais il est évident que pour l'auteur, ce n'est pas tant la victoire politique qui importe que la victoire de l'homme sur lui-même et sur le destin. Voici ce que dit Gaëtan Picon sur le titre de Malraux:

Que Malraux ait appelé L'Espoir le livre d'une défaite politique, voilà ce qui donne à ce titre sa vraie portée. Il s'agit de l'espoir absurde qui attache l'homme à on ne sait quel rêve de transformation métaphysique, et qui n'est rien d'autre que l'expression même de sa vitalité, de la force invincible qui le rend indiscernable de son souffle, confiance exaltée, et au plus profond des ténèbres, envers les puissances de l'homme qui témoignent de sa grandeur. (Picon, 1953: 78.) 
En face de ce livre, force est de reconnaître que nous sommes en présence d'un écrivain qui n'est pas un historien même si l'Histoire constitue la toile de fond de son œuvre. Si le titre constitue une déception, cette déception se précise et devient plus aiguë en analysant le roman sur le plan de la vérité historique.

Du point de vue historique, L'Espoir apparaît comme un roman raté. Même si le cadre géographique du roman est exact et rigoureux grâce à la connaissance de l'Espagne par Malraux, ce qui est raconté semble être une pure invention et imagination de l'auteur pour des commodités romanesques. Mais la question que nous sommes en droit de nous poser est celle de savoir si vraiment l'écrivain français voulait se laisser prendre à la magie de l'Histoire. Même si la guerre est présente dans le roman, elle ne l'est que du côté républicain auquel l'auteur accorde sa sympathie.

Pour l'écrivain, la vérité historique est travestie. Mais curieusement et paradoxalement, cette vérité historique travestie donne naissance à une autre beaucoup plus intéressante, à savoir, la vérité humaine, la vérité romanesque. Ce qui aurait dû être l'histoire de la guerre civile espagnole devient, contre toute attente, méditation sur la mort, sur le droit de tuer, sur la condition humaine.

Contrairement à l'historien dont le souci majeur est de faire revivre un événement historique dans toute sa réalité et son objectivité, le romancier-historien, sans pour autant méconnaître la valeur de l'événement en lui-même, s'intéresse plutôt à la relation entre l'individu et l'événement, au retentissement de cet événement en l'homme et à la manière dont il le perçoit. C'est dire que le romancier-historien, en reconstituant l'événement, fait partiellement le travail de l'historien mais il en vient même à le dépasser. Il donne une explication valable de l'Histoire. Grâce à Malraux, on comprend certains aspects de la guerre civile espagnole dont les historiens ont difficilement rendu compte: le problème du fascisme, de la foi chrétienne, les problèmes de la société espagnole. Il semble donc que le romancier-historien supplée l'historien là où ce dernier fait défaut. Leurs travaux sont complémentaires. Si l'Histoire est le reflet passif du réel chez l'historien, elle est plutôt réflexion objective chez le romancier. Même si le romancier-historien apparaît comme philosophe à travers sa méditation sur la condition humaine et les enseignements qu'il donne. En fait, il ne l'est pas. Il est plutôt artiste. Le roman semble donc être l'art de raconter des mensonges sur ce que vous savez pour finalement en faire quelque chose de plus réel que la réalité.

Il est donc évident que pour le romancier, la vérité n'est ni dans le fait ni dans l'événement historique, elle est dans l'art. La vérité romanesque et artistique prend le pas et l'emporte sur la vérité historique comme le confirme Hemingway sans équivoques:

La seule écriture valable, c'est celle qu'on invente, celle qu'on imagine. C'est ça qui rend les choses réelles. (Hemingway, 1999: 251.)

Contrairement à l'historien dont l'œuvre est essentiellement tournée vers le passé, Malraux semble beaucoup plus se préoccuper du présent et de l'avenir que du passé. 
En dernière analyse, c'est par son engagement que le romancier se démarque totalement de l'historien dont la rigueur, la passivité et l'objectivité excluent tout engagement. Si l'historien accorde peu ou presque pas de place à l'homme, le romancierhistorien, au contraire, est tout acquis à la cause humaine, à la condition humaine.

Forts de ces remarques, il semble que Malraux n'est pas un historien, qu'il n'est même pas un romancier-historien ; c'est un romancier tout court, un créateur, un artiste. Il veut tout simplement écrire un roman devenant de fait un historien du présent. L'auteur de La Condition humaine choisit des morceaux de l'Histoire et cible des événements types répondant à ses besoins. Il s'engage ainsi dans la perspective classificatoire et événementielle de la chronologie historique. Nous convenons avec Tzvetan Todorov que :

Le travail de l'historien, comme tout travail sur le passé, ne consiste jamais seulement à établir des faits mais aussi à choisir certains d'entre eux comme étant plus saillants et plus significatifs que d'autres, à les mettre ensuite en relation entre eux ; or ce travail de sélection et de combinaison est nécessairement orienté par la recherche, non de la vérité, mais du bien. (Todorov, 1995: 150).

La remarque faite au sujet du travail de l'historien s'applique fort bien à Malraux car même s'il n'écrit pas l'Histoire, il en présente au moins sa vision et sa lecture dans sa fiction. Il écrit dans une perspective historique. Certainement mu par le difficile devoir de mémoire, André Malraux se fait le romancier de l'Histoire pour affirmer sa personnalité.

\section{L'AFFIRMATION IDENTITAIRE}

Le caractère permanent et fondamental de Malraux semble difficile à déterminer. Moi haïssable de Pascal, insaisissable et mouvant de Bergson ou de Proust, hypnoses, aliénation, rêves, inconscient et subconscient, etc., la question de l'existence et de la nature du moi profond est au cœur des débats des années 1920. La question qu'on est en droit de se poser est celle de savoir si Malraux doit s'engager dans les affaires de L'Espagne. Daniel Bergez constate que:

L'Europe, comme l'individu lui-même, perd son identité. Dès lors, Malraux se tourne vers l'action. (Bergez, 2009: 299).

En fait, l'auteur de La Voie Royale cherche à se créer lui-même par ses propres choix. Les actes qu'il pose l'engagent et le mettent au devant de la scène. En 1926, Malraux justifie son attitude par le fait qu'il n'y a pas d'essence du moi profond, il n'y a de moi qu'en action, en situation ou en mouvement. L'essence de notre personnalité ne se concentre pas dans un secret figé dont nous devrions obtenir un jour la reconnaissance publique. L'attitude farfelue de Malraux, l'aventure sont autant de façons de laisser le moi en mouvement, de lui refuser la fixation dans une identité stable. Michel Raimond fait remarquer qu' «il semble que le goût du farfelu ait été une des constituantes de la personnalité d'André Malraux.» (Raimond, 1976: 207.) Et l'œuvre d'art, conçue sur un tel modèle, invite le moi à disparaître en elle, bien plus qu'à s'y révéler. Il n'est donc pas surprenant que Malraux ne puisse imaginer les héros de L'Espoir que comme des êtres privés de toute identité sociale reconnue - que cette reconnaissance prenne la 
forme de la considération bourgeoise, ou, à l'inverse, celle de l'humiliation. Tous les aviateurs internationaux du roman ont quitté leur pays, leur métier, leur famille pour participer à une guerre à portée universelle. Malraux fait observer qu'en:

(...) combattant avec les Républicains et les communistes espagnols, nous défendions des valeurs que nous tenions, que je tiens pour universelles. (Raimond, 1976: 219.)

Visiblement, c'est le début de la quête identitaire et spirituelle. Le romancier engage le combat contre le destin.

Il est temps, aux yeux de Malraux, déclare Brincourt, que l'individu s'ouvre à une réalité différente qui ne peut être qu'une réalité commune, universelle. (Brincourt, 1986: 30.)

Malraux rejette sans ambages le piège de l'individualisme où sombre l'Europe pour faire place à la solidarité. Son engagement pour la cause humaine est donc indéniable. S'inquiétant de la montée des fascismes dans le monde et sentant par conséquent la liberté de l'homme menacée, il se met aux côtés des Républicains. Il ne se contente pas seulement de tenir de beaux discours, il donne sa contribution financière pour les équiper militairement. Tout comme Hemingway, il est profondément antifasciste. C'est ainsi qu'il combat toutes les formes de tyrannie, d'oppression. Il veut restaurer l'homme dans sa dignité que méconnaissent les Fascistes.

Solidaire de ses camarades et anti-communiste en dépit du respect qu'il a vis-àvis de l'efficacité et de la discipline communiste, Magnin comme un métaphysicien est beaucoup plus enclin aux problèmes de la condition humaine qu'à la guerre.

L'Espoir n'éclaire délibérément les événements que d'un seul côté, celui des Républicains. Le témoignage, pris sur le vif, est rédigé à chaud, et ce roman est un livre de combat, celui d'un engagement partisan. Si l'on a, surtout dans une première lecture, une impression d'émiettement et de dispersion, c'est que le véritable héros de L'Espoir, ce n'est pas tel ou tel individu, c'est la révolution espagnole, c'est l'élan de tout un peuple. Pour Magnin, «la guerre sera longue...le peuple est optimiste. (Malraux, 1937: 134). L'action est multiple, et on le voit bien dès le début, où l'on passe constamment d'un lieu à un autre, d'un personnage à un autre, d'un point de vue à un autre l'unité étant toujours assurée par les lignes de force du livre, celle-ci en particulier: la nécessité de passer de l'insurrection spontanée à la guerre organisée. L'enthousiasme des Républicains, l'héroïsme d'actions isolées, ce n'est qu'une fête de la fraternité qui est, comme le dit Maurice Rieuneau, «condamnée à être écrasée ou à se transformer en organisation efficace.» (Raimond, 1988: 145.)

Idéologiquement, L'Espoir s'inscrit dans la perspective qui était alors celle du parti communiste : priorité absolue à l'effort de guerre, à la discipline, à l'organisation, avec tous les renoncements que ce choix implique. Cette thèse est toutefois nuancée par plusieurs éléments romanesques, une série d'entretiens, notamment, où les anarchistes s'interrogent sur la valeur d'une victoire obtenue en sacrifiant l'être au faire: «maintenant il ne s'agit plus d'être quelque chose, martèle René Lalou, mais de faire quelque chose.» (Gaillard, 1970: 77.)

D’une façon générale, en dépit du fait que $L^{\prime} E$ spoir soit un véritable roman de guerre et que Pour qui sonne le glas ne l'est presque pas, les mêmes thèmes fondamentaux 
se retrouvent aussi bien chez Malraux que chez Hemingway. Tout comme Pour qui sonne le glas, L'Espoir s'ordonne autour de l'engagement pour la cause, la condition humaine, la prise de position pour la dignité de l'homme, la solidarité et la fraternité virile, l'efficacité et la discipline communistes. C'est donc à juste titre que Roger Asselineau reconnaît que «l'œuvre de Hemingway rejoint celle de Malraux sur les plans thématique et idéologique». ( Asselineau, 1965: 19).

Une scène revient de façon obsédante dans le roman, celle d'un personnage attendant, impuissant, sa mort, probable ou effective. Cela veut dire que Malraux regarde la guerre de très près, non du côté de la gloire, de la bravoure et de l'héroïsme, mais du côté de la souffrance, de la mort et de la chair torturée, écorchée, lacérée au canon ou à la kalachnikov, des corps mutilés et sanguinolents.

La guerre est présente partout. Elle ne détruit pas seulement les corps, elle fragilise et ruine aussi l'individu. Celui-ci est un être éclaté, écartelé, disloqué dans ce qu'il a de plus profond, dans son essence même. C'est dire que l'univers de L'Espoir est un univers du pessimisme, de la désolation et de la mort. Si la torture est un thème récurrent dans $L^{\prime} E$ spoir, c'est parce qu'elle représente l'humiliation la plus extrême et la plus irréparable. Elle donne à ses victimes une identité proprement monstrueuse, sur laquelle plus aucune prise n'est possible. Mais l'humiliation fascine aussi tous les personnages.

Chez Malraux, l'œuvre d'art est toujours à l'opposé de la pente rêveuse, fantasmatique, morbide de l'imagination ; elle est le contraire de l'enlisement en soimême, elle est une négation de soi par dépassement, métamorphose. Les anarchistes de L'Espoir poussent jusqu'à l'absurde cette théorie du beau geste : il est, avec eux, la seule justification possible de la vie. L'action n'importe nullement par les résultats qu'elle obtient ou non, mais seulement par l'exemplarité du geste qui lui donne sa forme. Plutôt que de réaliser quelque chose dans sa quête identitaire, Malraux tient à offrir aux autres un modèle de vie, de mouvement, de dépassement, un style, une façon d'échapper aussi bien à l'établissement bourgeois qu'à la honte irréversible de l'humiliation. C'est la raison pour laquelle l'on peut considérer, L'Espoir comme roman reportage certes, mais roman de formation, d'apprentissage, «bildungsroman», roman existentiel, de la condition humaine, de l'absurde, de l'exemple.

Le risque couru alors par les anarchistes, on le voit notamment à Tolède lors du siège de l'Alcazar, c'est sombrer dans le spectaculaire - autre enlisement de l'identité, autre fixation d'une image de soi dans le regard des autres, non sans parenté, d'ailleurs, avec celle qui peut se produire sur la scène de l'humiliation.

C'est cette opposition entre l'enlisement et le dépassement que Jean-François Lyotard retrouve à sa manière lorsqu'il découvre chez Malraux ces deux pôles que constitue, selon lui, le «bios» et la «graphè»:

Dans l'espèce humaine comme dans les autres, la mort a pour agent le ventre de la reproduction, la femelle. Conséquemment, Malraux hait son temps d'enfance passé sous la tutelle des femmes. Silence de plomb sur la génération maternelle. La filiation virile qu'il oppose à celle-ci opère par la seule vertu de l'exploit, elle ne doit rien au bios. Les hommes ont en charge de transmettre 
l'énigmatique pouvoir de la graphè. [...] [E]st père, quel qu'il soit dans l'ordre biologique, celui qui tente le geste de résigner cet ordre et en transmet la trace. (Lyotard, 1996: 105).

Le thème du corps comme poids, souffrance ou fatigue est fréquent dans le roman. La conscience d'une filiation est au contraire ce qui rend possible la protestation virile contre cet ordre maternel, féminin, qui impose à chacun la lourde et peu malléable identité de son corps. L'importance de cette filiation virile explique le nombre élevé de pères - spirituels bien sûr car, comme le remarque Lyotard, un père ne saurait être naturel - qu'on retrouve dans L'Espoir: Garcia, Ximénes, Puig, Magnin. Car s'il faut un père aux héros de Malraux, c'est sans doute parce que le monde ne se présente jamais à la conscience d'un individu sans avoir été préparé, éclairé au préalable par une autre conscience.

La vie de Malraux a été une aventure, une prise de risques permanente qui s'est achevée dans la tragédie. La littérature du XXème siècle lui doit beaucoup. Il y a maintenant 36 ans (1976-2012) qu'il mourut tragiquement, âgé de 75 ans. Tandis que l'image du personnage public a commencé à pâlir, l'œuvre elle-même, longtemps obscurcie par les mythes qui entouraient son créateur, se détache avec plus de netteté. Au cours de sa vie agitée, Malraux joua tant de rôles - le journaliste, le ministre, l'homme politique - que l'on pouvait quelquefois oublier qu'il était avant tout un écrivain et que la seule chose qui, en définitive, comptât pour lui, c'était son œuvre. Et lorsqu'il sentit finalement sa puissance créatrice l'abandonner en même temps que ses forces physiques, il ne trouva plus aucune raison de continuer à vivre. Il rejoint fatalement sa propre philosophie selon laquelle:

Il est beau de mourir de sa mort, d'une mort qui ressemble à sa vie. Et mourir est passivité mais se tuer est acte. (Malraux, 1933: 239.)

Car son œuvre avait toujours constitué son unique défense contre ce sens du «nada», du néant, de l'absurde qui l'avait poursuivi tout au long des années.

Maintenant que les masques sont tombés, nous nous rendons compte qu'ils dissimulaient la figure sensible, d'une sensibilité même morbide, d'un artiste scrupuleux, tout consacré à son art.

Toute sa carrière est un témoignage de la condition de l'homme d'aujourd'hui. Désespérément conscient de sa solitude et de son impuissance dans un univers indifférent et chaotique, de l'homme malade de l'homme, depuis longtemps privé de Dieu et favorable au diable, Malraux essaye de forger un code moral qui lui assurerait tout au moins un endroit propre et bien éclairé afin de mener le combat contre les fatalités, qui encouragerait l'individu à se comporter avec grâce dans l'adversité, et l'aiderait à accepter l'horreur du néant avec stoïcisme et dignité.

Qui mieux que lui, pourrait-on s'interroger, a su créer son propre mythe, concourir à la fabrication de sa propre identité ou de sa propre légende? La question de l'identité, c'est celle de l'image publique sans laquelle Malraux n'aurait pas été lu jusqu'à ce jour. Le lecteur est frappé dans le roman par l'importance des valeurs de l'énergie, du courage, de la virilité des personnages que Malraux met en scène. 
Malraux permet, à travers l'écriture, à son énergie individuelle et identitaire de se déployer.

\section{CONCLUSION}

L'enjeu du présent essai a consisté à éclairer une double intention. Cette double intentionnalité procède de deux points névralgiques. Elle met d'abord en évidence l'utilisation consciente de l'Histoire comme substrat du roman entraînant par ce fait son exploitation pour aboutir à une fiction. C'est d'ailleurs ce que Jean-Marie Schaeffer appelle « la contamination du monde historique par le monde fictionnel.» (Schaeffer, 1999 : 141.) En réalité, c'est le monde imaginaire et imaginable qui s'empare du monde historique pour créer le monde fictionnel. Tel que posé, le monde fictionnel équivaut au monde imaginaire.

Dans cette idée qui figure la métaphore microbienne à travers le mot « contamination », il se dégage la notion forte d'altérité. Il en ressort que dans le rapport Histoire/fiction, la fiction corrode l'Histoire, la dénature et la dévisage pour la soumettre aux exigences de l'imagination. Là est tout le sens de l'exploitation et de l'esthétisation. Cependant, les traces flagrantes de l'Histoire perceptibles dans L'Espoir sont les marques de la référentialité qui accordent au roman un crédit de dénotation. Il y a donc interaction et interagissement entre ces deux disciplines et, par conséquent, l'action de la fiction n'est pas que dénaturation. L'ambiguïté relationnelle qui en découle a certainement motivé l'embarras de Paul Ricœur dans la désignation des rapports entre Histoire et fiction. Il parle invariablement et inversement de «fictionnalisation de l'histoire » et d' « historicisation de la fiction.»(Ricœur, 1985: 331.)

Malraux est à la une parce qu'il réécrit l'Histoire ou reproduit la réalité et la vérité de l'époque. Ce qui est en fait l'enjeu de notre essai, c'est le rapport entre réalité et fiction d'une part et le rapport reportage et fantasmation d'autre part. L'apport ou la présence écrasante de l'histoire dans L'Espoir démontre que Malraux sert une image mutilée de la condition humaine particulièrement inhumaine $d^{\prime}$ un $X X^{\text {ème }}$ siècle effroyable.

Si le souci de la popularité et/ou de l'enjeu pécuniaire l'emporte sur l'ambition de retracer avec rigueur et fidélité les faits du passé, le pas à franchir entre l'Histoire et le roman n'existe plus. Cela voudrait dire que l'historien ne se réfèrera plus à l'objectivité et à la rigueur des faits qu'il présente mais il créera ses vérités. Car la fiction créatrice et imaginaire sera conforme à cette histoire inventée pour plaire ou pour propager des mythes de dévalorisation ou de surévaluation de tel ou tel peuple ou de tel ou tel fait. Malgré l'ambiguïté profonde qui régit le discours historique quant à sa véracité, un postulat apparent le qualifie de véridique. C'est probablement ce flou qui pousse Malraux à s'inspirer des faits historiques pour écrire sa fiction. Au-delà de cette hypothèse $a$ priori trop facile, il se pose le crucial problème de la réécriture de l'Histoire. 
Il faut réécrire l'Histoire parce que les faits ne se sont pas toujours déroulés comme ils sont racontés par les historiens. Les multiples témoignages aussi différents les uns des autres - rapportant un même fait - sont très éloquents à ce sujet. Les déconvenues et imbroglios se font jour quant à la version à authentifier ou à croire. Le romancier donne lui aussi sa lecture de l'Histoire. Malraux comme le chasseur, a éventré l'Histoire pour extraire les tripes sanguinolentes sujet de son écriture. Il plonge sa plume au cœur des souffrances que la guerre a généré et le sang qui lui sert d'encrier retrace avec sagacité les tourments et les dérives humaines. Les guerres à répétition de par le monde, les dictatures renaissantes, les nostalgies morbides en sont une vibrante illustration.

Pour tout dire, l'une des principales conclusions ou l'essentiel de ce à quoi on a abouti dans l'argumentation est que comme dans toute création littéraire, la réalité et la fiction font concurrence. Les deux notions sont présentes dans L'Espoir au point où il est difficile d'établir la frontière de séparation. Malraux montre ici que l'Histoire est nourrie prioritairement par la réalité sans toutefois négliger la fantasmation qui appartient à la fiction ou à l'imagination créatrice du romancier.

Peu importe que Malraux aie quitté le cours des événements avant la fin de l'histoire. Il avait joué à fond son rôle dans l'amour comme à la guerre. Il a été inspiré par cette dernière à travers $L^{\prime} E$ spoir qui, outre ses qualités intrinsèques, est créateur d'un genre nouveau, le roman de guerre:

Ce genre, affirme Jean Labesse, devait connaître une grande fortune, non seulement dans les pays belligérants, mais dans le monde entier. (Labesse, 1995: 161).

Pourquoi ce succès mondial? Malraux a su exprimer la grandeur et surtout l'horreur de l'homme au XXème siècle qu'il a contribué à façonner et dont il s'est, à d'autres égards, contenté d'observer le déroulement mais en acteur, sur la scène même, et non pas en spectateur dans la salle. «L'homme est la somme de ses actes» conclut-il. (Malraux, 1933: 191.)

À ce titre, il figure à juste mesure parmi les lauréats des prix Goncourt ( $\mathrm{La}$ Condition humaine), Louis Delluc (L'Espoir) qui ont su montrer à quoi peut servir la littérature ou mieux encore, à quoi peut servir un écrivain. Et c'est à juste titre que Boisdeffre affirme que:

Si Malraux avait prêché l'engagement, ce n'était pas seulement par des mots mais aussi par l'exemple, en homme pour qui les idées ne sont pas faites pour être pensées, mais vécues. (Boisdeffre, 1963: 26.)

Aujourd'hui, avec plus de soixante seize ans de recul, le voyage de Malraux à Madrid en juillet 1936 conserve toute sa valeur humaine. À l'époque, les Espagnols ne s'y éprirent pas. Ils virent en Malraux un lutteur de marque, venu parmi eux à un moment de grande crise non seulement pour donner l'appui de sa solidarité morale et l'inspiration spirituelle de sa présence, mais aussi pour être le symbole de quelque chose de plus vaste, en un mot d'universel.

Du point de vue littéraire, l'importance du séjour de Malraux en Espagne vient en grande partie du fait que ce qu'il vit, apprit et sentit outre-Pyrénées lui permit de 
donner aux premiers chapitres de son roman ce ton d'histoire authentique et vécue qui a tant frappé les critiques. Heureusement, grâce au talent de Malraux, ce moment rare et émouvant - qui est à la source de son engagement en Espagne - fut capté pour toujours dans une œuvre d'art : son grand roman, L'Espoir. Il est donc probable, selon les termes de Jacques Madaule, «que cette guerre, quelle qu'en soit l'issue, sera à l'Espagne ce que fut à la France la Révolution: c'est-à-dire la promotion du peuple». (Gaillard, 1970: 83.)

\section{BIBLIOGRAPHIE}

ARON, Raymond. (1984) : Paix et guerre entre les nations, Paris, Calmann-Lévy.

ASSELINEAU, Roger. (1965): The Literary reputation of Hemingway in Europe, Paris, Lettres Modernes.

BARTHES, Roland et alii. (1977): Poétique du récit, Paris, Le Seuil.

BEAUMARCHAIS, Jean-Pierre (De) et alii. Dictionnaire des écrivains de langue française, Paris, Larousse/VUEF, 2001, t1 et 2.

BERGEZ, Daniel, Précis de littérature française. (2009): Paris, Armand Colin.

BIET, Brighelli, Rispail. (1987) : Malraux la création d'un destin, Paris, Gallimard, coll.

«Découvertes».

BOISDEFFRE, Pierre (De). (1963): Les Écrivains français d'aujourd'hui, Paris,

P.U.F., coll. «Que sais-je?».

BOURNEUF, Roland et OUELLET, Réal. (1972) L'Univers du roman, Paris, P.U.F., Littératures Modernes.

BRECHON, Robert, La Condition humaine d'André Malraux. (1972) Paris, Hachette, 1972. BRINCOURT, André, Malraux le malentendu. (1986): Paris, Grasset et Fasquelle.

CAMUS, Albert. (1947): La Peste, Paris, Gallimard.

FITCH, Brian, T. (1964) Les Deux univers romanesques d'André Malraux, Paris, Minard.

FUENTES, Carlos. (1997): Géographie du roman, Paris, Gallimard.

GAILLARD, Pol. (1970): Les Critiques de notre temps et Malraux, Paris, Garnier.

GENETTE, Gérard. (1999): Figures IV, Paris, Le Seuil.

HEMINGWAY, Ernest. (1999): Nouvelles complètes, Paris, Gallimard.

HEMINGWAY, Ernest. (1944): Pour qui sonnele glas, Paris, Le Livre de Poche. Traduction de Denise Van Ayme (Pseudonyme de temps de guerre de Denise Van Moppès). LABESSE, Jean. (1995) : Les Grandes expressions littéraires au XX ${ }^{\text {ème }}$ siècle, Paris, Ellipses/ éditions marketing S.A.

LACASSE, Rodolphe. (1977): Hemingway-Malraux-destin de l'homme, Paris, Cosmos.

LANGLOIS, Walter. (1973): André Malraux 2, visages du romancier, Paris, Lettres Modernes.

LARRAT, Jean-Claude. (2001): André Malraux, Paris, Librairie Générale Française. 
LE PELLEC, Yves. (1991): Rocard, Marcienne, « avant-propos » in Caliban (le roman historique), Presses Universitaires du Mirail, Toulouse.

LYOTARD, Jean-François. (1996): Signé Malraux, Paris, Grasset.

MALRAUX, André. (1937): L'Espoir, Paris, Gallimard, coll. « Folio ».

MALRAUX, André. (1967): Le Miroir des limbes II La Corde et les souris, Paris, Gallimard, coll. «Folio».

MALRAUX, André. (1948): Les Noyers de l'Altenburg, Paris, Gallimard.

MALRAUX, André. (1933): La Condition humaine, Paris, Gallimard.

MARION, Denis. (1996): Le Cinéma selon André Malraux, Paris, Cahiers du cinéma.

MAURIAC, François.(1972): Le Romancier et ses personnages, Paris, R-A. Corrêa, Le Livre de poche.

PICON, Gaëtan. (1953): Malraux par lui-même, Paris, Le Seuil, coll. «Écrivains de toujours».

RAIMOND, Michel. (1988): Le Roman, Paris, Armand Colin.

RAIMOND, Michel. (1976): Le Signe des temps, Paris, CDU et SEDES.

RICEUR, Paul. (2001) : Histoire et vérité, Paris, Le Seuil.

RICEEUR, Paul. (1985) : Temps et récit, Tome III, Paris, Le Seuil.

SCHAEFFER, Jean-Marie.(1999): Pourquoi la fiction ?, Paris, Le Seuil.

STENDHAL, Le Rouge et le noir. (1988): Paris, Jean Claude Lattès, (1830).

STEPHANE, Roger. (1996): Malraux, premier dans le siècle, Paris, Gallimard, coll. « Les Cahiers de la N.R.F.».

TODOROV , Tzvetan. (1987): Les Abus de la mémoire, Paris, Arléa. 